SUBJECT AREAS:

EVOLUTION

NEUROSCIENCE

ANIMAL BEHAVIOUR

Received

7 March 2014

Accepted

21 May 2014

Published

13 June 2014

Correspondence and requests for materials should be addressed to A.W. (aw2@standrews.ac.uk)

\section{Synchrony and motor mimicking in chimpanzee observational learning}

\author{
Delia Fuhrmann ${ }^{1,2}$, Andrea Ravignani ${ }^{3}$, Sarah Marshall-Pescini ${ }^{1,4}$ \& Andrew Whiten ${ }^{1}$
}

${ }^{1}$ Centre for Social Learning and Cognitive Evolution, School of Psychology \& Neuroscience, University of St Andrews, KY 16 9JP, St Andrews, Scotland, ${ }^{2}$ Institute of Cognitive Neuroscience, Division of Psychology and Language Science, University College London, WCIN 3AR, London, England, ${ }^{3}$ Department of Cognitive Biology, University of Vienna, 1090 Vienna, Austria, ${ }^{4}$ Comparative Cognition, Messerli Research Institute, University of Veterinary Medicine, Vienna, Medical University of Vienna, University of Vienna, Vienna, Austria.

Cumulative tool-based culture underwrote our species' evolutionary success, and tool-based nut-cracking is one of the strongest candidates for cultural transmission in our closest relatives, chimpanzees. However the social learning processes that may explain both the similarities and differences between the species remain unclear. A previous study of nut-cracking by initially naive chimpanzees suggested that a learning chimpanzee holding no hammer nevertheless replicated hammering actions it witnessed. This observation has potentially important implications for the nature of the social learning processes and underlying motor coding involved. In the present study, model and observer actions were quantified frame-by-frame and analysed with stringent statistical methods, demonstrating synchrony between the observer's and model's movements, cross-correlation of these movements above chance level and a unidirectional transmission process from model to observer. These results provide the first quantitative evidence for motor mimicking underlain by motor coding in apes, with implications for mirror neuron function.

T he last decade has seen a substantial growth in our understanding of the evolution of social learning, traditions and cultural transmission over the course of hominin evolution and in animal behaviour more generally ${ }^{1,2}$. However, it is our distinctive human capacity for the building of cumulative cultural acquisitions, from technology to language, that has become well recognised as one of the keys to the peculiar success and global distribution of our species ${ }^{3,4}$. A widespread view is that one of the key factors that underwrite this capacity lies in the human facility for high fidelity imitative copying of the actions of others. This is contrasted with forms of social learning in even our closest relatives, chimpanzees, which appear to be more often limited to replicating only the environmental results of others' actions. This process is referred to as emulation ${ }^{3-5}$. There is evidence that the action imitation that characterises humans is underwritten not merely by the perceptual coding of what others do, but by motor coding of the kind now associated with the functioning of mirror neurons ${ }^{6,7}$. This has been argued to be a distinctive, evolved human development, contrasting with a lack of an imitative function for the mirror neuron system first identified in monkeys 6 .

Whether chimpanzees and other apes are indeed essentially emulators, or have some human-like imitative capacities, has been a focus of much controversy fuelled by contrasting experimental findings $\mathrm{s}^{5,8}$. Moreover, little is known of the nature and function of the mirror neuron systems of apes; the neuroimaging that primarily provides such evidence in humans is impractical for apes, and the invasive approach on which the discovery of mirror neurons in monkeys was based is avoided for both ethical and practical reasons. Here, we report an analysis of fortuitous and remarkable behaviour in a chimpanzee involved in our experimental studies on the social learning of nut-cracking skills, that we believe casts light on the nature of social learning in our closest relatives (the imitation versus emulation debate ${ }^{5,8}$ ) and on the likely role of mirror neurons in apes in such contexts.

Chimpanzee nut-cracking is one of the most sophisticated tool-use behaviours known in non-human primates $^{9,10}$. It is also one of the best examples of culturally transmitted behaviours in the wild as it exhibits clear regional variation. Nut-cracking occurs only in West and Central Africa, but not in East Africa ${ }^{11-13}$. Notably, its distribution renders genetic, environmental and asocial learning explanations improbable ${ }^{14,15}$.

The acquisition of nut-cracking has been described as "education by master-apprenticeship"16. Information is thought to be transmitted unidirectionally - mostly from mother to infant ${ }^{17}$. Wild chimpanzees appear to exhibit a critical period for the learning of nut-cracking between 3 and 7 years of age ${ }^{17}$. This is reminiscent of the windows of opportunity for learning that exist in humans, particularly with regards to the acquisition of complex skills like language ${ }^{18}$. 
A first controlled experimental investigation of the cultural nature of nut-cracking was provided by Marshall-Pescini and Whiten (2008) who conducted a study with 12 wild-born, East-African, sanctuaryliving chimpanzees between 2 and 6 years of age. The chimpanzees were split into an experimental group and control group. The experimental group was given the opportunity to observe a model demonstrate nut-cracking over five trials. The control group received access only to nuts, anvils and hammers. While no chimpanzee in the control group was able to crack nuts over the five trials, 3 out of 4 chimpanzees older than 3 years of age in the experimental group learned the behaviour. All participants older than 3 years of age were able to learn nut-cracking in the follow-up phase, where both experimental and control group observed additional demonstrations.

Of particular import for the present study, incidental observations of real-time mimicking of cracking actions were recorded for three young chimpanzees in the experimental group ${ }^{9}$ (Supplementary Videos 1-5). While an expert model cracked nuts with a stone hammer, episodes of similar rhythmic hitting motions that appeared to echo these were recorded seven times in Baluku (Figure 1) and once in Umugenzi. Additionally, Ikuru engaged in rhythmic whole-body rocking motions. Both Ikuru and Umugenzi also occasionally placed a hand and sometimes a foot on the experimenter's hitting arm. This behaviour has been termed co-action in other social learning experiments and has been suggested to provide kinaesthetic feedback of the demonstrators' actions ${ }^{19}$.

These observations are of substantial interest in light of controversies about whether bodily imitation is part of the chimpanzee's social learning repertoire at all ${ }^{5,8}$. It has been suggested that chimpanzee culture in general ${ }^{5,20}$, and nut-cracking specifically ${ }^{17}$, might instead rely on emulation alone. As noted above, the term emulation describes copying the results of a model's actions whereas imitation refers to copying the actions themselves ${ }^{21}$. Recently, evidence has accrued from other social learning studies that chimpanzees ${ }^{22}$ and other non-human primates ${ }^{23,24}$ may copy others' actions, matching the body part with which they have seen another individual solve a task.

A distinction is often drawn here between mimicking and imitation. Mimicking is defined by the minimal criterion of exhibiting a motor match to the movements of another individual ${ }^{25}$, whereas the ascription of imitation typically demands additional criteria, such as that the action is done with a similar goal to the original, and/or possesses a degree of novelty, neither of which are requirements for mere mimicking ${ }^{21}$. Both imitation and mimicking may be exemplified in synchrony between the behaviour of two individuals, synchrony being defined in the Oxford English Dictionary as "coincidence or agreement in point of time"26. A special case of synchrony which is of relevance to the present case is entrainment, which describes the spatiotemporal coordination between indivi- duals, often in relation to rhythmic signals. In humans, synchronisation is ubiquitous and may occur in tapping ${ }^{27}$, rocking ${ }^{28}$ and facial mimicry ${ }^{29}$. In our species, such synchrony appears to facilitate social bonding $^{30}$ and joint action ${ }^{31}$. There is also a burgeoning interest in synchronisation in non-human animals. Motor matching to auditory and visual aspects of behaviour patterning has been reported in several species, including a sulphur-crested cockatoo ${ }^{32}$, California sea $\operatorname{lion}^{33}$ and (to a limited extent) rhesus macaques ${ }^{34,35}$. Notably, a recent study with Japanese macaques showed that these animals may influence temporal features of each other's movements in a laboratory setting ${ }^{36}$.

A motor matching phenomenon that has received particular interest recently is automatic imitation ${ }^{37}$, where task-irrelevant motion patterns facilitate congruent, and impede incongruent, responses. The best evidence for this phenomenon is provided by studies on humans ${ }^{27,37,38}$. It has been proposed that the proximate function of automatic imitation may lie in the social acquisition of relatively opaque behaviours, while its ultimate functionality may be the facilitation of high-fidelity cultural transmission ${ }^{39}$. At present, it remains unclear, however, how automatic imitation relates to spontaneous motor mimicking and there is a vivid debate as to whether these processes are supported by a shared representation of perception and action in the mirror neuron system ${ }^{37,40,41}$

Mirror neurons, cerebral units responsive during both action observation and execution, were originally discovered in macaque monkeys ${ }^{42,43}$ and electrophysiological evidence of their existence has since been provided for humans as well ${ }^{44}$. Mirror Neurons have been proposed to mediate several aspects of human social cognition including theory of mind ${ }^{45}$, action understanding and imitation ${ }^{46}$. It remains unclear how the "mirroring" properties of these neurons arise and what their exact properties and functions are ${ }^{47}$. It is puzzling, for example, that monkeys, for which the best evidence of mirror neurons exist, are generally not apt to imitate and even training monkeys to copy actions has often failed. There is much more solid evidence of imitation in apes but neither direct (e.g. electrophysiological) or indirect (e.g. fMRI) evidence for the existence of MNs has been obtained for apes ${ }^{48}$ for practical and ethical reasons.

In the light of these controversies, the videos from the MarshallPescini and Whiten experiment (2008), containing some of the only documentations of apparent motor mimicking in apes, have been met with interest and were cited as evidence for inter-individual action coding in chimpanzees. It has been suggested, for example, that the motor behaviour exhibited in the video materials is functional in that it provides the learner with kinaesthetic feedback ${ }^{49}$, is consistent with direct inter-individual action coding at a motor program level ${ }^{9,41,49}$, indicative of bodily imitation ${ }^{8,50}$, related to interindividual synchronisation observable, for example, in bird flocks, and linked to mirror neuron systems ${ }^{51}$. a

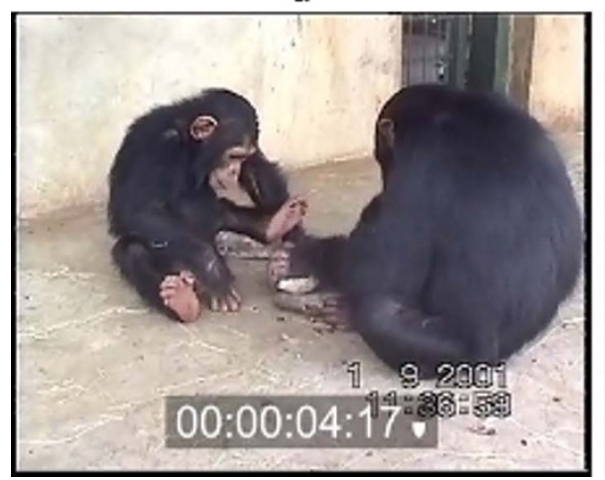

b

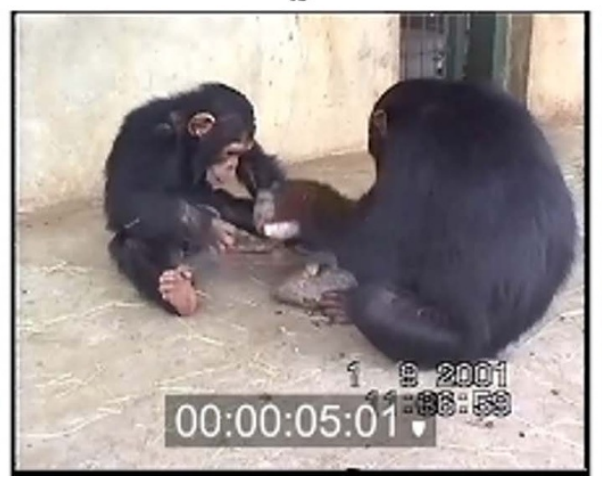

c

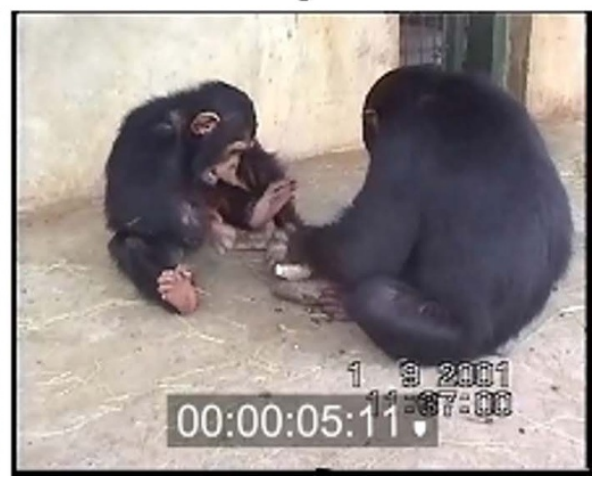

Figure 1 Baluku observing Mawa. Sequence of stills (a, b, c) from Video 1 showing Baluku (left) as the observer and Mawa (right). Mawa is holding a stone in his left hand and using it to crack a nut in front of him. Baluku is moving his right hand without a stone in his hand or a nut to crack. 

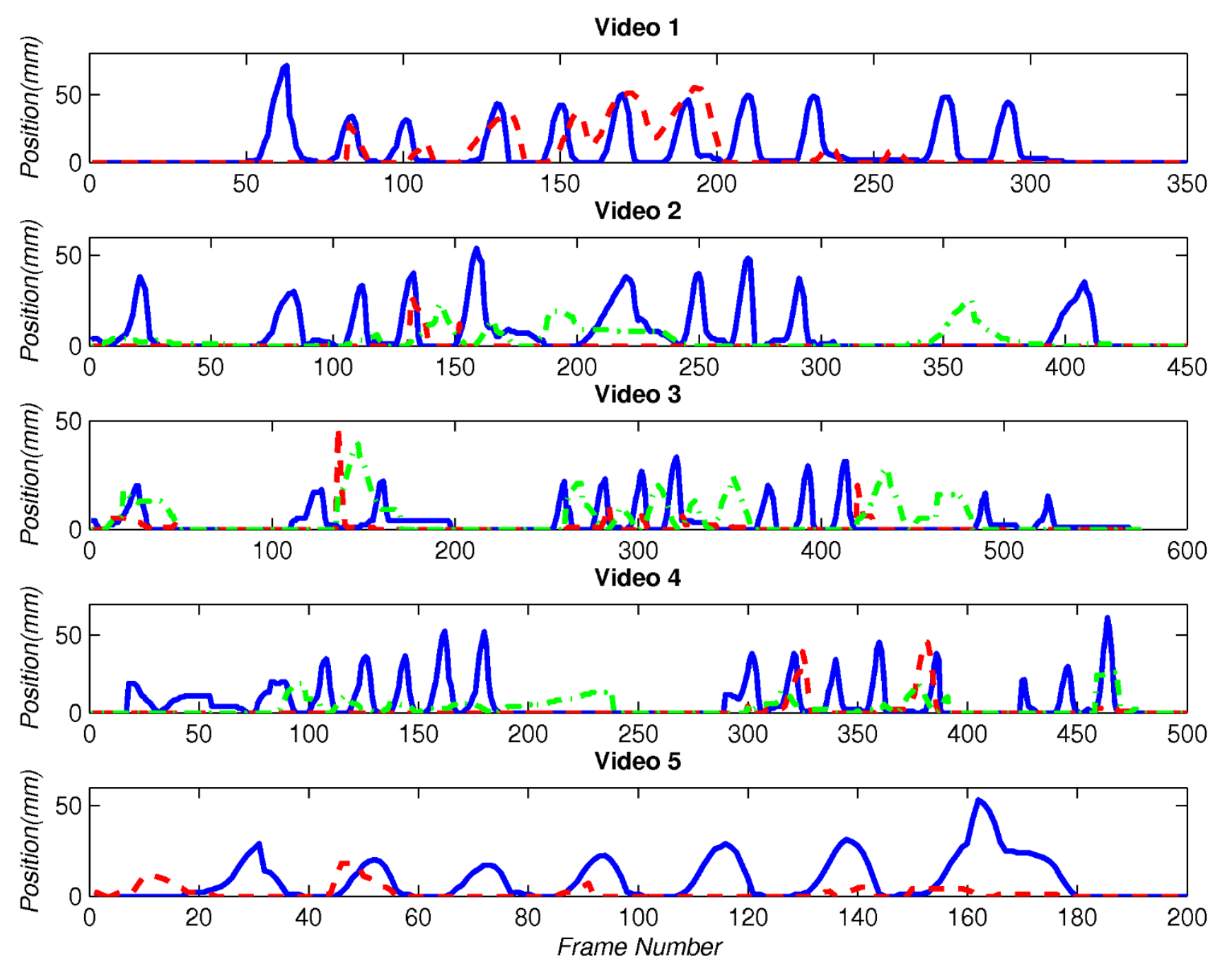

Figure $2 \mid$ Graphs of the movement of models and observers. The movement was coded as the position on screen over the frames of the video and is plotted only for episodes of nut-cracking for the model and attention to nut-cracking for the observer. Details of Video 1: Model Mawa (blue, solid line) and Observer Baluku (red, dashed line - hand), Video 2: Mawa (blue, solid line) and Baluku (red, dashed line- hand, green, dash-dot line- back), Video 3: Mawa (blue, solid line) and Baluku (red, dashed line - hand, green, dash-dot line - ear), Video 4: Model Experimenter (blue, solid line) and Observer Baluku (red, dashed line- hand, green, dash-dot line -ear), Video 5: Experimenter (blue, solid line) and Baluku (red, dashed line: hand).

In order to test the proposition that the action sequences filmed provide evidence of inter-individual action representation in apes, an empirical approach to the information contained in the videos is here explored. Three questions in particular are investigated:

1.) Are the model's and observer's movements synchronous?

2.) Is the correlation between the movements likely to have arisen by chance?

3.) Is there evidence of a transmission process between individuals and was information transmitted unidirectionally from the model to the observer?

\section{Results}

The videos contained a varying number of nut-cracking and mimicking bouts which consisted of beats or sways (Supplementary Table 1; Supplementary videos 1-5). The movement of the model's and observer's body parts described quasi-periodic waveforms during nut-cracking and mimicking (Figure 2).

Comparison of interval durations. The interval durations, calculated as time elapsed between contiguous maxima in body position (corresponding to "peaks" in Figure 2), were not normally distributed according to the Shapiro-Wilk test in all but one condition (Supplementary Table 2). The medians and interquartile ranges of the interval durations of the models and observers in Videos 1-5 are displayed in Figure 3.

Pairwise Kolmogorov-Smirnoff (K-S) tests showed no significant differences between the distributions of the interval durations of the model and observer in any of the five videos $(\mathrm{p}>0.05)$. This indicates that the patterns of movement of the model and observer had similar durations, i.e. for each video the action durations of model and observer were drawn from a common underlying distribution (See Table 1.)

Monte Carlo permutation test of cross-correlations. After differencing all time-series once, the series were covariance-stationary (CS) according to the ADF and KPSS test in all but one series, where the ADF, but not KPSS test indicated a violation (Supplementary Table 3). A Monte Carlo permutation test showed that withinvideo cross-correlations were significantly greater $(p<0.05)$ than between-videos cross-correlations, indicating that the cross-correlation between the models and observers within videos is unlikely to have arisen by chance. See Table 2 . 


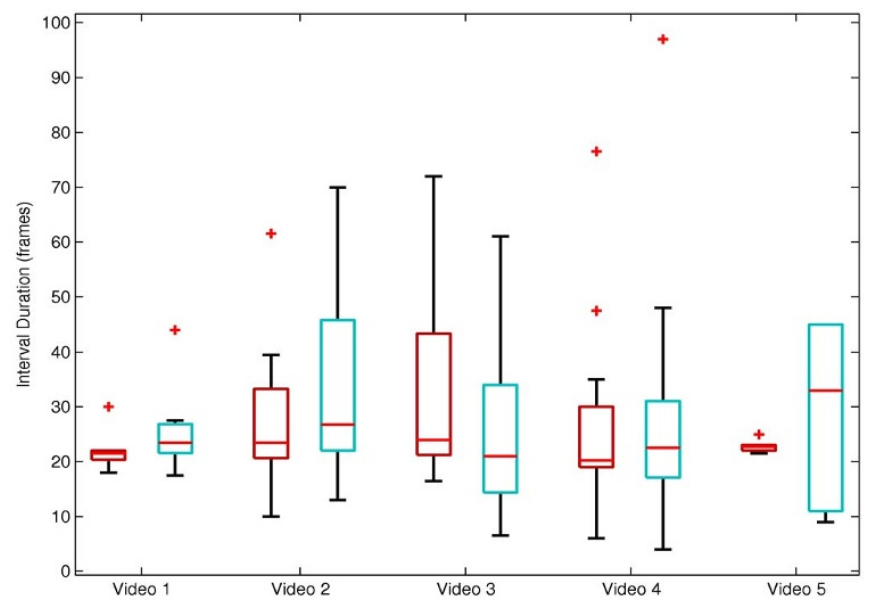

Figure 3 Boxplots of the interval durations displaying the medians and interquartile ranges of the models (red) and observers (green) in Videos 1-5.

Granger Causality Analysis (GCA). Due to insufficient data points, the optimal model order could not be determined reliably for Videos 3 and $4^{52}$. These Videos were not further analyzed. For Videos 1, 2 and 5 the model actions significantly Granger-caused the observer actions and not vice versa $(\mathrm{p}<0.05)$, as hypothesized (Table 3$)$. The results of Video 2 should be interpreted with caution, however, as the model consistency and the adjusted sums of squares of the observer were relatively low.

\section{Discussion}

These results provide the first quantitative evidence for motor mimicking and action entrainment between apes. We refer to mimicking rather than imitation here because Baluku, whilst being in a social learning context, did not have a physical goal - he had no anvil and no nut as a target, nor was he holding a hammer stone. Neither can we ascertain whether the action (hammering) was novel to him. By entrainment we mean that there was temporal synchrony between the model's and the observer's beating actions ${ }^{31}$, and in this, it was the observer, Baluku, who followed, or entrained, on the actions of model. These results are largely consistent with the proposal that the videos document inter-individual action representation in this social learning context ${ }^{41,49,51,53}$. In the following discussion we consider each of the analyses supporting these conclusions in more detail.

The interval durations of the hitting motions of the model and observer did not differ within videos, indicating that the movements were synchronous. This finding provides empirical evidence for Ferrari et al.'s (2009) suggestion that the videos evidence inter-individual synchronisation in apes. It is further consistent with previous studies on spontaneous synchronisation in other mammals and birds documenting spontaneous and flexible adjustment of their own movements to audio, visual and social stimuli ${ }^{32,34-36}$. Nonetheless, the observational findings reported here deserve to be complemented with experimental evidence in the future. For example, spontaneous

Table 1 | The results of the K-S Test (D) test comparing the interval durations of the model and observer within videos. P-values above 0.05 indicate that the distributions of the interval durations did not differ significantly from one another

\begin{tabular}{cccccc} 
& VIDEO 1 & VIDEO 2 & VIDEO 3 & VIDEO 4 & VIDEO 5 \\
\hline$D$ & 0.460 & 0.217 & 0.429 & 0.255 & 0.667 \\
$p$ & 0.375 & 0.913 & 0.080 & 0.621 & 0.139 \\
\hline
\end{tabular}

Table 2 | Results of the Cross-Correlation Analysis and Monte Carlo permutation test. Abbreviations: maximum cross-correlation at lag $x$ (maximum CC), pseudo-p-value (1-tailed) for the crosscorrelation simulation ( $p$-value)

\begin{tabular}{|llcclc} 
& VIDEO 1 & VIDEO 2 & VIDEO 3 & VIDEO 4 & VIDEO 5 \\
\hline maximum CC & 0.218 & 0.195 & 0.394 & 0.259 & 0.221 \\
lag & 5 & 5 & 2 & 4 & 28 \\
$p$-value & 0.024 & $<0.001$ & $<0.001$ & 0.016 & 0.024 \\
\hline
\end{tabular}

synchronisation during nut-cracking could be studied in apes in a controlled laboratory setting, like that explored by Nagasaka and colleagues $^{36}$.

The Monte Carlo permutation we conducted indicates that crosscorrelation of the movements within videos was unlikely to have arisen by chance. This finding dovetails with the notion that the videos show inter-individual action coding ${ }^{49,53}$. This Monte Carlo resampling method was found to provide a useful and powerful tool for the investigation of correlational data ${ }^{54,55}$ and the method can therefore be recommended for future studies on spontaneous mimicking.

Despite the power of these analytical techniques, we acknowledge that an unavoidable limitation of our study is the sample size. For practical and ethical reasons, sample sizes in studies on synchronicity in nun-human animals are often small and studies of single individuals are not unusual ${ }^{32}$. What is more, few chimpanzees we studied spontaneously acted in the way described here and this is perhaps an inherent aspect of the phenomenon. There are reasons to believe that Baluku might be unusual and more apt to copy others than most apes. A recent study of social learning in apes, for example, found that Baluku succeeded in imitation tasks that all other chimpanzees in the study failed ${ }^{50}$.

Perhaps relevant to this finding is that in general, downstream inhibition prevents motor coding by mirror neurons from generating unbridled mimicking of others, yet in humans there may nevertheless be minor 'leakage' overcoming such inhibition, when mimetic actions are manifested in identification with others' actions, as in

Table 3 | Results of the Granger Causality Analysis. The F-statistic for the GCA and corresponding $p$-value for the column variable causing the other variable in the same video are displayed. The AIC is reported, as well as tests of covariance-stationarity (CS). 0 indicates CS for AIC, 1 indicates CS for KPSS. Further, the adjusted sums of squares and model consistency are reported. Potential problems in the model consistencies are indicated by sums of squares values $<0.3$ and consistencies $<80 \%$. Abbreviations: Model (M), Observer (O)

\begin{tabular}{|c|c|c|c|c|c|c|c|c|c|}
\hline \multicolumn{2}{|l|}{ VIDEO 1} & \multicolumn{2}{|c|}{ VIDEO 2} & \multicolumn{2}{|c|}{ VIDEO 3} & \multicolumn{2}{|c|}{ VIDEO 4} & \multicolumn{2}{|c|}{ VIDEO 5} \\
\hline$M$ & O & $M$ & O & $M$ & 0 & $M$ & 0 & $M$ & 0 \\
\hline $\begin{array}{l}\boldsymbol{A} / \boldsymbol{C} \\
22 \\
\mathbf{A D F}\end{array}$ & & 5 & & & - & & & 29 & \\
\hline $\begin{array}{l}\text { ADF } \\
0 \\
\text { KPSS }\end{array}$ & 0 & 0 & 0 & 0 & 0 & 1 & 1 & 0 & 0 \\
\hline F-value & 1 & 1 & 1 & 1 & 1 & 0 & 1 & 1 & 1 \\
\hline $\begin{array}{l}3.939 \\
\text { p-value }\end{array}$ & 1.084 & 3.628 & 1.142 & - & - & - & - & 1.902 & 1.380 \\
\hline $\begin{array}{l}<0.001 \\
\text { Adiustec }\end{array}$ & $\begin{array}{l}0.368 \\
\text { d residu }\end{array}$ & $\begin{array}{l}0.003 \\
\text { ual sum }\end{array}$ & $\begin{array}{c}0.338 \\
\text { ns of so }\end{array}$ & uar & - & - & - & 0.014 & 0.133 \\
\hline 0.537 & $\begin{array}{r}0.565 \\
\text { onsisteı }\end{array}$ & $\begin{array}{c}0.406 \\
\text { ncy (\%) }\end{array}$ & 0.218 & - & - & - & - & 0.699 & 0.475 \\
\hline 69.731 & & 42. & 831 & & & & & 83. & .060 \\
\hline
\end{tabular}


everyday activities like watching boxing or other sports that happen to intensely engage the observer; extreme forms of this are displayed in the condition of echopraxia ${ }^{56}$. Baluku appeared to be a relatively rare chimpanzee exhibiting the mild 'spill-over' of mimetic actions seen in everyday human contexts. There are observational indications that other chimpanzees might at times engage in similar behaviour $^{9,10}$ but this has not been investigated empirically so far. A systematic survey of mimicking behaviour in wild chimpanzee populations for which there is evidence for cultural variation similar to Whiten and colleagues ${ }^{13}$ may afford more clarity.

The Granger Causality analysis showed that, for the three out of five videos where Granger Causality analysis could be conducted, information was transmitted from Model to Observer but not vice versa. This provides empirical evidence consistent with the unidirectional account of the transmission of nut-cracking behaviour ${ }^{16,17}$. Granger Causality analysis proved indispensable for this study. It is singular in its scope in that it is able to provide empirical insights into the directionality of transmission processes. Moreover, it provides a statistical approximation to causality when correlational data cannot be extended to causal manipulations. Therefore, it is recommended for future studies on social transmission.

Our findings are consistent with the proposal that Baluku engaged in bodily mimicking 9 . They are also in line with reports on body-part matching in the course of social transmission in chimpanzees ${ }^{22}$ and other non-human primates ${ }^{23,24}$. Moreover, they suggest that chimpanzee nut-cracking does not necessarily have to be purely emulative $^{17}$, but could be supported by a wider repertoire of social learning processes involving bodily matching. In this case, emulation cannot provide an account because the observer chimpanzee was not recreating a result - no hammer or nut were involved, only the beating action.

In line with this spectrum of bodily copying, mirror neurons have also been found to vary in their specificity. In the macaque brain, strictly congruent mirror neurons that code for an exact match between observation and action are only half as common as broadly congruent neurons. Whiten predicted that based on the match of this ratio to the frequency of emulation vs imitation in macaques, human brains should have a higher proportion of strictly versus broadly congruent MNs, while apes should be intermediate ${ }^{48}$.

We think that it remains unclear if the stringent criteria for 'process imitation' (copying novel action structures) can be considered satisfied $^{8,50}$. Nut-cracking as such was novel to Baluku prior to the study, as confirmed by the fact that he showed no nut-cracking behaviours when he was provided with nuts and tools in the baseline trials in the original experiment ${ }^{9}$. The act of hitting something with an object was likely not new to him, however. We also note that after observing the model, he had begun to try to crack nuts before producing the mimetic actions analysed in the present paper. It thus remains unclear whether he was engaged in process imitation, or perhaps 'contextual imitation', in which what is socially learned is the application of a known action to a novel context.

In conclusion, we have reported systematic analyses of fortuitous actions consistent with motor mimicking of the kind now identified with mirror neuron function, a finding of importance across many fields of investigation, from brain science to comparative analyses of social learning, the evolution of cultural transmission and related phenomena such as empathy ${ }^{7}$. Future experiments will need to provide direct, causal, experimental evidence for the proposal that chimpanzees code witnessed actions motorically. One of the strongest indicators of such inter-individual action coding to date is automatic imitation ${ }^{37}$ which has been tested successfully with interference tasks in humans ${ }^{27}$. In such tasks the production of movements incongruent with those of a partner is usually found to be hampered compared to the production of congruent motion ${ }^{27,37}$. The findings of the present study could be complemented by testing whether automatic imitation of movements such as rocking ${ }^{28}$ or hitting, similar to nut-cracking, occurs in chimpanzees, too. To gain a better understanding of the ontogenetic and phylogenetic origins of automatic imitation it would also be instructive to investigate how the phenomenon develops in children.

\section{Methods}

Materials. The videos analysed here were obtained from the video records of a previous experiment on social learning of nut-cracking ${ }^{9}$. The authors of this study surveyed the records for incidental observations of mimicking and digitised five episodes. These episodes were revisited for the purpose of the present study.

Participants. The five videos analysed here feature Baluku as the observer. Baluku is a male chimpanzee who was 3-years old at the time of the experiment. Over different demonstration trials he observed either the experimenter, Sarah Marshall-Pescini, or the 5-year-old chimpanzee Mawa, crack nuts (Supplementary Table 1).

Procedure. The video videos were imported into the motion graphics program Adobe ${ }^{\circledR}$ After Effects ${ }^{\circledR}$. The Stabilize Motion function was applied to compensate for the camera shake of the hand-held camcorder. Afterwards, a grid consisting of thin red lines was overlaid on the focal area of the video to aid orientation during measurements. Then, a point on the hitting hand of both the model and observer was chosen for coding (Table 1). The choice was made based on salience and visibility. For Videos 2-4, where the hand of the observer was temporarily obscured, an additional body part was coded (Table 1). This body part was again selected based on salience and visibility. The position of the body part was coded on a frame-by-frame basis and measured as the closest distance to the lower side of the frame. In Video 4, the movement of the observer's ear was horizontal. Therefore the position was coded as the closest distance to the left side of the frame.

General data pre-processing. For Video 1 and Video 5 the coding procedure resulted in two time series each - one coding the position of the model's hand and one the position of the observer's. Three time series were obtained for Video 2-4 as an additional body part of the observer was coded. For Videos $2-4$, the time series coding the movement of the hand are displayed in Figure 2 but were not further analysed due to an insufficient number of data points. To standardize the start and end of the time series across videos, the series were truncated so that each series began with the onset of the model's first nut-cracking bout and ended when the observer stopped attending to the model. The data of the observer in Video 5 required additional processing as the observer's hand was obscured by the model's arm for several episodes and no additional body part was suitable for coding. The MATLAB function inpaint_nans, Method 4 (D'Errico, J., inpaint_nans, http://www.mathworks.co.uk/matlabcentral/ fileexchange/4551-inpaint-nans (2012), (Date of access: 09/05/2014)) was used to interpolate the missing values from the incomplete time series. The function uses partial differential equations to interpolate holes in arrays.

Data analysis. Three different statistical approaches were implemented. In order to test the hypothesis that the model's and observer's movements were synchronous, a comparison of the interval durations (a) was conducted. To investigate whether the correlation between the movements was likely to have arisen by chance, a Monte Carlo permutation test of cross-correlations was conducted (b). Granger Causality Analysis (c) was used to assess whether there is evidence of a transmission process between individuals and to test if information was transmitted unidirectionally from the model to the observer.

\section{a) Comparison of the interval durations}

The interval durations were defined as the number of frames between consecutive maxima in each time series. A prerequisite for synchrony of the model's and observer's motions would be that the interval durations are sufficiently similar. The Kolmogorov-Smirnov Test (K-S Test) was used to compare the distributions of the intervals of the model and observer within each video. Similar to other studies on synchronisation in non-human primates ${ }^{34}$, it was hypothesised that, due to synchrony, there would be no difference between the model and observer within videos.

b) Monte Carlo permutation test of cross correlations

Intervals of the time series during which the observer was not attending to the model and thus no opportunity for mimicking was given, were excluded from the following steps of the analysis. This concerned only Videos 2-4 where observation was interrupted (Table 1). Using the MATLAB Granger Causality Connectivity Analysis (GCCA) toolbox ${ }^{55}$, the time series' were checked for covariance-stationarity using the $\mathrm{ADF}^{52}$ and KPSS test ${ }^{57}$. If the time series were non-CS, they were differenced using the function cca_diff and checked for CS again. Note that the differenced data reflects the changes in the original time series over time ${ }^{55}$

A Monte-Carlo permutation test of cross-correlations at different lags ("time shifts" between two time series) was performed using a custom Python script (www.python.org). The function calculates the maximum cross-correlation between the model and observer within each video and then compares it to the cross-correlation of all possible between-video pairings of models and observers (for a range of lag values). To perform the Monte Carlo permutation test, shorter time series were padded with zeros up to the length of the longest time series. Similar to other studies on matching behaviour in animals ${ }^{32}$, it was 
hypothesised that if the observer tried to synchronise with the model (instead of "resonating" to a fixed internal frequency, like in dogs wagging their tail), the natural cross-correlation would be significantly greater than the simulated (between-videos) cross-correlations.

c) Granger Causality Analysis (GCA)

The time series were then analysed for Ganger Causality. The concept was originally developed for Economics and has been recently applied to functional Neuroimaging data ${ }^{55}$. According to GCA a signal $X_{1}$ G-causes a signal $X_{2}$ if the past information from $X_{1}$ and $X_{2}$ is better at predicting $X_{2}$ than the information contained in the past values of $X_{2}$ alone. Similarly, we hypothesized that if movements of the model $X_{1}$ had an influence on the observer $X_{2}$ (but not vice versa), the model's time series would G-cause the observer but the observer would not G-cause the model. Note that G-causality is thus conceptualised to capture some, but not all aspects of physical causality ${ }^{58}$.

The GCCA toolbox function cca_granger_regress was used to carry out the GCA. The model order (i.e. the number of time-lags to include) was estimated via the $\mathrm{AIC}^{59}$. This was obtained from the $\mathrm{R}$ function VARselect in the package Vars. The model validity was checked via the model consistency and residual sums of squares provided by the function cca_granger_regress.

1. Laland, K. N. \& Galef, B. G. (eds.). The Question of Animal Culture (Harvard University Press, Cambridge, 2009).

2. Whiten, A., Hinde, R. A., Laland, K. N. \& Stringer, C. B. Culture evolves. Philos. Trans. R Soc. Lond. B 366, 938-948 (2011).

3. Tomasello, M. The Cultural Origins of Human Cognition (Harvard University Press, Cambridge, 1999).

4. Boyd, R., Richerson, P. J. \& Henrich, J. The cultural niche: why social learning is essential for human adaptation. Proc. Natl. Acad. Sci. USA 103, 1-8 (2011).

5. Tennie, C., Call, J. \& Tomasello, M. Ratcheting up the ratchet: on the evolution of cumulative culture. Philos. Trans. R Soc. Lond. B 364, 2405-2415 (2009).

6. Rizzolatti, G., Fogassi, L. \& Gallese, V. Neurophysiological mechanisms underlying the understanding and imitation of action. Nature Rev. Neurosci. 2, 661-670 (2001).

7. Iacobini, M. in The Primate Mind: Built to Connect with Other Minds. 32-47 (Harvard University Press, Cambridge, 2012).

8. Whiten, A., McGuigan, N., Marshall-Pescini, S. \& Hopper, L. M. Emulation, imitation, over-imitation and the scope of culture for child and chimpanzee. Philos. Trans. R Soc. Lond. B 364, 2417-2428 (2009).

9. Marshall-Pescini, S. \& Whiten, A. Social learning of nut-cracking behavior in East African sanctuary-living chimpanzees (Pan troglodytes schweinfurthii). J. Comp. Psychol. 122, 186-194 (2008).

10. Whiten, A. et al. Cultures in chimpanzees. Nature 399, 682-685 (1999).

11. Matsuzawa, T., Humle, T. \& Sugiyama, Y. The Chimpanzees of Bossou and Nimba (Springer Verlag Publishing, Tokyo, 2011).

12. McGrew, W. C. Culture in nonhuman primates? Annu. Rev. Anthropol. 27, 301-328 (1998).

13. Morgan, B. J. \& Abwe, E. E. Chimpanzees use stone hammers in Cameroon. Curr. Biol. 16, R632-R633 (2006).

14. Byrne, R. W. Culture in great apes: using intricate complexity in feeding skills to trace the evolutionary origin of human technical prowess. Philos. Trans. $R$ Soc. Lond. B 362, 577-585 (2007).

15. Boesch, C., Marchesi, P., Marchesi, N., Fruth, B. \& Joulian, F. Is nut cracking in wild chimpanzees a cultural behaviour? J. Hum. Evol. 26, 325-338 (1994).

16. Matsuzawa, T. et al. in Primate Origins of Human Cognition and Behavior (Springer-Verlag Publishing, New York, 2001).

17. Biro, D. et al. Cultural innovation and transmission of tool use in wild chimpanzees: evidence from field experiments. Anim. Cogn. 6, 213-223 (2003).

18. Kuhl, P. K. Brain mechanisms in early language acquisition. Neuron 67, 713-727 (2010).

19. Horner, V., Whiten, A., Flynn, E. \& Waal, F. B. M. d. Faithful replication of foraging techniques along cultural transmission chains by chimpanzees and children. Proc. Natl. Acad. Sci. USA 103, 13878-13883 (2006).

20. Tennie, C., Call, J. \& Tomasello, M. Evidence for emulation in chimpanzees in social settings using the floating peanut task. PLoS ONE 5, e10544 (2010).

21. Whiten, A., Horner, V., Litchfield, C. \& Marshall-Pescini, S. How do apes ape? Learn. Behav. 32, 36-52 (2004).

22. Buttelmann, D., Carpenter, M., Call, J. \& Tomasello, M. Enculturated chimpanzees imitate rationally. Dev. Sci. 10, F31-F38 (2007).

23. van de Waal, E. \& Whiten, A. Spontaneous emergence, imitation and spread of alternative foraging techniques among groups of vervet monkeys. PLoS ONE 7, e47008 (2012)

24. Voelkl, B. \& Huber, L. True imitation in marmosets. Anim. Behav. 60, 195-202 (2000).

25. Tomasello, M., Kruger, A. C. \& Ratner, H. H. Cultural learning. Behav. Brain Sci. 16, 495-552 (1993)

26. Oxford University Press. Oxford English Dictionary: Synchrony, <http://www. oed.com/view/Entry/196403? redirectedFrom $=$ synchrony\# eid $>$ (2014), (Date of access: 09/05/2014).

27. Brass, M., Zysset, S. \& von Cramon, D. Y. The inhibition of imitative response tendencies. Neuroimage 14, 1416-1423 (2001).
28. Richardson, M. J., Marsh, K. L., Isenhower, R. W., Goodman, J. R. \& Schmidt, R. C. Rocking together: dynamics of intentional and unintentional interpersonal coordination. Hum. Mov. Sci. 26, 867-891 (2007).

29. Sato, W. \& Yoshikawa, S. Spontaneous facial mimicry in response to dynamic facial expressions. Cognition 104, 1-18 (2007).

30. Oullier, O., de Guzman, G. C., Jantzen, K. J., Lagarde, J. \& Kelso, J. A. S. Social coordination dynamics: measuring human bonding. Soc. Neurosci. 3, 178-192 (2008).

31. Sebanz, N., Bekkering, H. \& Knoblich, G. Joint action: bodies and minds moving together. Trends Cogn. Sci. 10, 70-76 (2006).

32. Patel, A. D., Iversen, J. R., Bregman, M. R. \& Schulz, I. Experimental evidence for synchronization to a musical beat in a nonhuman animal. Curr. Biol. 19, 827-830 (2009).

33. Cook, P., Rouse, A., Wilson, M. \& Reichmuth, C. A California sea lion (Zalophus californianus) can keep the beat: motor entrainment to rhythmic auditory stimuli in a non vocal mimic. J. Comp. Psychol. 127, 412-427 (2013).

34. Zarco, W., Merchant, H., Prado, L. \& Mendez, J. C. Subsecond timing in primates: comparison of interval production between human subjects and rhesus monkeys. J. Neurophysiol. 102, 3191-3202 (2009).

35. Merchant, H., Zarco, W., Pérez, O., Prado, L. \& Bartolo, R. Measuring time with different neural chronometers during a synchronization-continuation task. Proc. Natl. Acad. Sci. 108, 19784-19789 (2011).

36. Nagasaka, Y., Chao, Z. C., Hasegawa, N., Notoya, T. \& Fujii, N. Spontaneous synchronization of arm motion between Japanese macaques. Nature Sci. Rep. 3, (2013).

37. Heyes, C. Automatic imitation. Psychol. Bull. 137, 463-483 (2011).

38. Leighton, J., Bird, G., Orsini, C. \& Heyes, C. Social attitudes modulate automatic imitation. J. Exp. Soc. Psychol. 46, 905-910 (2010).

39. Heyes, C. M. \& Ray, E. D. What Is the significance of imitation in animals? Adv. Stud. Behav. 29, 215-245 (2000).

40. Heyes, C. Evolution, development and intentional control of imitation. Philos. Trans. R Soc. Lond. B 364, 2293-2298 (2009).

41. Shea, N. Imitation as an inheritance system. Philos. Trans. R Soc. Lond. B 364, 2429-2443 (2009).

42. Gallese, V., Fadiga, L., Fogassi, L. \& Rizzolatti, G. Action recognition in the premotor cortex. Brain 119, 593-609 (1996).

43. di Pellegrino, G., Fadiga, L., Fogassi, L., Gallese, V. \& Rizzolatti, G. Understanding motor events: a neurophysiological study. Exp. Brain Res. 91, 176-180 (1992).

44. Mukamel, R., Ekstrom, A. D., Kaplan, J., Iacoboni, M. \& Fried, I. Single-neuron responses in humans during execution and observation of actions. Curr. Biol. 20, 750-756 (2010).

45. Gallese, V. \& Goldman, A. Mirror neurons and the simulation theory of mindreading. Trends. Cogn. Sci. 2, 493-501 (1998).

46. Rizzolatti, G. \& Craighero, L. The mirror-neuron system. Annu. Rev. Neurosci. 27, 169-192 (2004).

47. Heyes, C. Where do mirror neurons come from? Neurosci. Biobehav. Rev. 34 , 575-583 (2010)

48. Whiten, A. Monkeys, apes, imitation and mirror neurons. Cortex 49, 2941-2943 (2013).

49. de Waal, F. B. M. \& Ferrari, P. F. Towards a bottom-up perspective on animal and human cognition. Trends Cogn. Sci. 14, 201-207 (2010).

50. Tennie, C., Call, J. \& Tomasello, M. Untrained chimpanzees (Pan troglodytes schweinfurthii) fail to imitate novel actions. PLos One 7, e41548 (2012).

51. Keysers, C. Primate cognition: copy that. Nature 482, 158-159 (2012).

52. Hamilton, J. D. Time series analysis. (Princeton University Press, Princton, 1994)

53. Ferrari, P. F., Bonini, L. \& Fogassi, L. From monkey mirror neurons to primate behaviours: possible 'direct' and 'indirect' pathways. Philos. Trans. $R$ Soc. Lond. $B$ 364, 2311-2323 (2009).

54. Preacher, K. J. \& Hayes, A. F. Asymptotic and resampling strategies for assessing and comparing indirect effects in multiple mediator models. Behav. Res. Meth. 40, 879-891 (2008).

55. Seth, A. K. A MATLAB toolbox for Granger causal connectivity analysis. J. Neurosci. Meth. 186, 262-273 (2010).

56. Brass, M., Ruby, P. \& Spengler, S. Inhibition of imitative behaviour and socia cognition. Philos. Trans. R Soc. Lond. B. 364, 2359-2367 (2009).

57. Kwiatkowski, D., Phillips, P. C. B., Schmidt, P. \& Shin, Y. Testing the null hypothesis of stationarity against the alternative of a unit root: how sure are we that economic time series have a unit root? J. Econometrics 54, 159-178 (1992).

58. Granger, C. W. J. Testing for causality: a personal viewpoint. J. Econ. Dyn. Control 2, 329-352 (1980).

59. Akaike, H. A new look at the statistical model identification. IEEE T. Autom. Contr. 19, 716-723 (1974).

\section{Acknowledgments}

We thank David Christensen for his assistance with the video editing and Josep Call, Tecumseh Fitch and Tetsuro Matsuzawa for helpful discussions of the materials presented here. A.R. was supported by ERC Advanced Grant 230604 SOMACCA to W. Tecumseh Fitch. SMP was supported by funding from the European Research Council under the European Union's Seventh Framework Programme (FP/2007-2013)/ERC Grant Agreement n. [311870]. 


\section{Author contributions}

S.M.P. and A.W. collected the video material we analysed here and designed the original study. D.F. conducted the video coding. D.F. and A.R. analysed the data. D.F., A.R., S.M.P. and A.W. wrote the manuscript.

\section{Additional information}

Supplementary information accompanies this paper at http://www.nature.com/ scientificreports

Competing financial interests: The authors declare no competing financial interests.
How to cite this article: Fuhrmann, D., Ravignani, A., Marshall-Pescini, S. \& Whiten, A. Synchrony and motor mimicking in chimpanzee observational learning. Sci. Rep. 4, 5283; DOI:10.1038/srep05283 (2014).

(c) (i) $\Theta$ This work is licensed under a Creative Commons Attribution-NonCommercialNoDerivs 4.0 International License. The images or other third party material in this article are included in the article's Creative Commons license, unless indicated otherwise in the credit line; if the material is not included under the Creative Commons license, users will need to obtain permission from the license holder in order to reproduce the material. To view a copy of this license, visit http:// creativecommons.org/licenses/by-nc-nd/4.0/ 